\title{
TWO-SCALE PRODUCT APPROXIMATION FOR SEMILINEAR PARABOLIC PROBLEMS IN MIXED METHODS
}

\author{
Dongho Kim, Eun-Jae Park, and Boyoon Seo
}

\begin{abstract}
We propose and analyze two-scale product approximation for semilinear heat equations in the mixed finite element method. In order to efficiently resolve nonlinear algebraic equations resulting from the mixed method for semilinear parabolic problems, we treat the nonlinear terms using some interpolation operator and exploit a two-scale grid algorithm. With this scheme, the nonlinear problem is reduced to a linear problem on a fine scale mesh without losing overall accuracy of the final system. We derive optimal order $L^{\infty}\left((0, T] ; L^{2}(\Omega)\right)$-error estimates for the relevant variables. Numerical results are presented to support the theory developed in this paper.
\end{abstract}

\section{Introduction}

In order to efficiently compute a number of integrals involving the coefficients or nonlinear terms arising in the variational formulation of the differential equations, one uses approximation of the coefficients, e.g., the interpolation, the projection or the quadrature formulas of the coefficients. The use of product approximation of the coefficients in Galerkin finite element methods for nonlinear problems has been analyzed by several authors (e.g., $[8,28,7,12,17])$. They applied the interpolation operator to the nonlinear coefficients and obtained error estimates of optimal convergence order with much less computational effort.

Chen and Douglas [5] analyzed the approximation of coefficients in mixed finite element method for nonlinear parabolic problems in the continuous-intime setting. There, the authors suggested two examples for the approximation of coefficients, namely, projection of the nonlinear coefficients into finite element

Received March 25, 2013; Revised August 6, 2013.

2010 Mathematics Subject Classification. Primary 65K10, 65M12, 65M60.

Key words and phrases. two-scale grid, product approximation, interpolation of coefficients, mixed finite element method, semilinear parabolic problem.

This research was supported by Basic Science Research Program through the National Research Foundation of Korea (NRF) funded by the Ministry of Education, Science and Technology NRF-2012R1A2A2A01046471. 
spaces and evaluation of the integral involving nonlinear term by a quadrature rule. However, in computing the discrete solution for the semilinear parabolic model problem under consideration, their schemes can be complicated from the implementation point of view.

In our paper, we propose elementwise interpolation of the nonlinear coefficients in mixed finite element methods for semilinear parabolic equations. This leads to great computational savings in obtaining matrix systems at every time step. Since the reduced system obtained by interpolation of the nonlinear term is yet nonlinear, it still requires an efficient solver for the resulting nonlinear algebraic equations on fine-scale mesh. For this, we introduce a two-scale method based on finite element spaces with grids of two different scales.

The two-scale method was first introduced by $\mathrm{Xu}[31,32]$ with the Galerkin method for semilinear and nonlinear elliptic boundary value problems. A new discretization technique, based on finite element spaces defined on two scales of different sizes, is presented. The author suggests an efficient algorithm and gives an error analysis. In the linear but not symmetric positive-definite (SPD) case, the idea of two scales is used to reduce the problem to an SPD. This is done by solving a non-SPD problem on a much smaller space. For nonlinear problems, the standard finite element method is used on the coarsest mesh. This solution is used as a starting value for the iterative solution on the finer mesh. Optimal accuracy is obtained even if the ratio of the coarse to the fine mesh is large.

Since the pioneering work of $\mathrm{Xu}$, the two-scale method was further investigated by many authors. For instance, Bi and Ginting [1] have applied this method to finite volume element method for linear and nonlinear elliptic problems. Layton and Lenferink [18] have also studied this method for Navier-Stokes equations. Recently, Jin, Shu and Xu [16] have applied the two-grid idea to decouple systems of partial differential equations.

On the other hand, mixed finite element methods have been successfully applied to several areas of interest, in particular, fluid flows in porous media $[14,27,29,22]$. Linear and nonlinear second order elliptic problems are studied in $[25,11,3,26,23,24,19,20]$. Two-scale mixed methods for parabolic problems are studied in references $[10,30,6]$. In particular, Dawson and Wheeler [10] have applied two-grid method combined with mixed finite element method to parabolic problems with nonlinear diffusivity. There, the fully nonlinear system is solved on a coarse scale of size $H$. The nonlinearities are expanded about the coarse scale solution, and the resulting linear but nonsymmetric system is solved on a finescale of size $h$. Error estimates are derived to demonstrate $\mathcal{O}\left(\Delta t+h^{k+1}+H^{2 k+1}\right)$ convergence with standard mixed finite element spaces of order $k$. Later, Wu and Allen [30] have applied two-grid scheme to mixed finite element method for reaction-diffusion equations with nonlinear reaction and obtained the error bound $\mathcal{O}\left(\Delta t+h^{k+1}+H^{2 k+2}\right)$. But a gap is found in the proof of Theorem 3.2 in the paper [30]. The proof requires $L^{4}(\Omega)$ error 
estimate in order to bound quadratic terms arising from linearization. Moreover, the authors of the paper [6] essentially fixed this gap using the properties of superconvergence. However, Lemma 4.1 in their paper should be modified due to the use of the inverse estimate and Lemma 4.2 is valid under the space and time mesh restriction $\Delta t \cdot H^{-1}=\mathcal{O}(1)$. Therefore, their main theorem (Theorem 4.1) should be modified accordingly.

Aforementioned issues are incorporated into our paper. Moreover, from the implementation point of view, our scheme is much simpler than that of [6] mainly due to the elementwise interpolation of the nonlinear coefficients. Consequently, we derive error estimates bounded by $\mathcal{O}\left(\Delta t+h^{k+1}+H^{2 k+1}\right)$ for $k \geq 1$ without losing overall accuracy of the final system on fine scale mesh. Note that we show the error bound $\mathcal{O}\left(\Delta t+h+H^{2}\right)$ for the lowest index $k=0$. In order to improve coarse scale convergence for higher $k$, we propose an interpolation operator based on Gaussian points. Then, we demonstrate error estimates $\mathcal{O}\left(\Delta t+h^{k+1}+H^{2 k+2}\right), k=1,2$, using the interpolation operator based on some Gaussian nodal points on triangular mesh.

Now, consider the following semilinear parabolic model problem:

$$
\left\{\begin{aligned}
u_{t}-\operatorname{div}(a(x) \nabla u) & =f(u) & & \text { in } \Omega \times J, \\
u & =0 & & \text { on } \partial \Omega \times J, \\
u(\cdot, 0) & =u^{0} & & \text { on } \Omega,
\end{aligned}\right.
$$

where $\Omega \subset \mathbb{R}^{2}$ is a convex planar domain with smooth boundary $\partial \Omega, J:=(0, T]$ and $0<\beta_{0}^{-1} \leq a(x) \leq \alpha_{0}^{-1}$.

Let $V:=\overline{L^{2}}(\Omega)$ and $\boldsymbol{M}:=H(\operatorname{div} ; \Omega)$, where the space

$$
H(\operatorname{div} ; \Omega):=\left\{\boldsymbol{\sigma} \in\left[L^{2}(\Omega)\right]^{2}: \operatorname{div} \boldsymbol{\sigma} \in L^{2}(\Omega)\right\} .
$$

Let $(\cdot, \cdot)$ denote the $L^{2}(\Omega)$-inner product and given an integer $m \geq 0, W^{m, p}(\Omega)$ denote the usual Sobolev space provided the norm

$$
\|v\|_{m, p}:=\left\{\sum_{|\beta| \leq m}\left\|D^{\beta} v\right\|_{p}^{p}\right\}^{1 / p}
$$

and semi-norm

$$
|v|_{m, p}:=\left\{\sum_{|\beta|=m}\left\|D^{\beta} v\right\|_{p}^{p}\right\}^{1 / p}
$$

When $p=2$, we denote $W^{m, p}(\Omega)$ by $H^{m}(\Omega)$ and write $\|v\|_{m}:=\|v\|_{m, 2}$. If $m=0$ we usually write $\|v\|:=\|v\|_{0}$. Furthermore, for finite element $K$ we use the notation $\|v\|_{m, p, K}$ and $|v|_{m, p, K}$.

We present the regularity assumptions which are sufficient to obtain required estimates. 
(A1) The $f(u)$ is a smooth function of $u \in \mathbb{R}$, i.e., there exists a bound $B_{1}$ such that

$$
\left|f^{(i)}\right| \leq B_{1}, \quad i=0,1,2, \ldots
$$

(A2) If $u$ is the solution of (1), there exists a constant $B_{2}$ such that

$$
\|u\|_{L^{2}\left(J ; H^{k+1}(\Omega)\right)}+\left\|\frac{\partial u}{\partial t}\right\|_{L^{2}\left(J ; H^{k+1}(\Omega)\right)}+\left\|\frac{\partial^{2} u}{\partial t^{2}}\right\|_{L^{2}\left(J ; L^{2}(\Omega)\right)} \leq B_{2},
$$

where

$$
\|w\|_{L^{2}(J ; X)}:=\left(\int_{0}^{T}\|w(\cdot, t)\|_{X}^{2} d t\right)^{1 / 2} .
$$

We note that under the hypotheses of the theorems to follow, our approximations converge uniformly to $u$; thus (A1) actually needs hold only in a neighborhood of the solution (c.f., [13]).

The rest of this paper is organized as follows. In Section 2, we describe our two-scale schemes combined with interpolation of coefficients for mixed finite element method. In Section 3, we derive $L^{\infty}\left(J ; L^{2}(\Omega)\right)$-error estimates for the scheme which show the optimal order of convergence on fine scale mesh. Finally, in Section 4, we present numerical results which confirm the theory developed in this paper.

\section{Description of the methods}

Let $\boldsymbol{\sigma}:=-a(x) \nabla u$ and $\alpha:=a(x)^{-1}$, then problem (1) has the following variational form: find $(u, \boldsymbol{\sigma}) \in V \times \boldsymbol{M}$ such that

$$
\begin{aligned}
(\alpha \boldsymbol{\sigma}, \boldsymbol{\tau})-(u, \operatorname{div} \boldsymbol{\tau}) & =0, \quad \forall \boldsymbol{\tau} \in \boldsymbol{M}, \quad \forall t \in J, \\
\left(\frac{\partial u}{\partial t}, v\right)+(\operatorname{div} \boldsymbol{\sigma}, v) & =(f(u), v), \quad \forall v \in V, \quad \forall t \in J, \\
u(\cdot, 0) & =u^{0}, \quad \text { in } \Omega .
\end{aligned}
$$

Let $\mathcal{T}_{h}$ be a family of quasi-uniform partition of $\Omega$ into triangles or rectangles of diameter not greater than $h$. Boundary finite elements are allowed to have one curvilinear edge or side. Associated with $\mathcal{T}_{h}$, we take standard mixed finite dimensional subspaces $\boldsymbol{M}_{h} \subset \boldsymbol{M}$ and $V_{h} \subset L^{2}(\Omega)$, for example, the RaviartThomas space $R T_{k}$ of order $k$.

If we use the backward Euler scheme for discretization with respect to the time variable it follows from (3) and (2) that for $n=1, \ldots, N$,

$$
\begin{aligned}
\left(\alpha \boldsymbol{\sigma}^{n}, \boldsymbol{\tau}\right)-\left(u^{n}, \operatorname{div} \boldsymbol{\tau}\right) & =0, \quad \forall \boldsymbol{\tau} \in \boldsymbol{M}, \\
\left(\frac{u^{n}-u^{n-1}}{\Delta t}, v\right)+\left(\operatorname{div} \boldsymbol{\sigma}^{n}, v\right) & =\left(f\left(u^{n}\right), v\right)+\left(e^{n}, v\right), \quad \forall v \in V,
\end{aligned}
$$

where $\Delta t$ is time step size, $t_{n}:=n \Delta t, u^{n}(\cdot):=u\left(\cdot, t_{n}\right), \boldsymbol{\sigma}^{n}(\cdot):=\boldsymbol{\sigma}\left(\cdot, t_{n}\right)$, and $e^{n}:=\frac{u^{n}-u^{n-1}}{\Delta t}-\frac{\partial u^{n}}{\partial t}$ is the truncation error associated with the backward Euler 
difference approximation. The space-time fully discrete problem is defined as follows: for $n=1, \ldots, N$, find $\left(u_{h}^{n}, \boldsymbol{\sigma}_{h}^{n}\right) \in V_{h} \times \boldsymbol{M}_{h}$ such that

$$
\begin{gathered}
\left(\alpha \boldsymbol{\sigma}_{h}^{n}, \boldsymbol{\tau}\right)-\left(u_{h}^{n}, \operatorname{div} \boldsymbol{\tau}\right)=0, \quad \forall \boldsymbol{\tau} \in \boldsymbol{M}_{h} \\
\left(\frac{u_{h}^{n}-u_{h}^{n-1}}{\Delta t}, v\right)+\left(\operatorname{div} \boldsymbol{\sigma}_{h}^{n}, v\right)=\left(f\left(u_{h}^{n}\right), v\right), \quad \forall v \in V_{h} .
\end{gathered}
$$

The following estimates are well known results (c.f., [21]): for each time level $n$, there exists positive constant $C$, independent of $h$ but dependent on function $u$ such that

$$
\begin{aligned}
\left\|u^{n}-u_{h}^{n}\right\| & \leq C\left(\Delta t+h^{r}\right), & 1 \leq r \leq k+1, & k \geq 0, \\
\left\|\boldsymbol{\sigma}^{n}-\boldsymbol{\sigma}_{h}^{n}\right\| & \leq C\left(\Delta t+h^{r}\right), & 1 \leq r \leq k+1, & k \geq 0 .
\end{aligned}
$$

Now, we define an operator $Q_{h}$ to interpolate the nonlinear term $f\left(u_{h}\right)$. For any element-wise continuous function $v$, i.e., $\left.v\right|_{K} \in C(\bar{K})$ for each element $K \in \mathcal{T}_{h}$, let $Q_{h}$ be the operator which maps $f(v)$ to $Q_{h} f(v) \in V_{h}$ defined by

$$
Q_{h} f(v)(\xi):=f(v(\xi))
$$

at each nodal point $\xi$. Then we have well-known approximation property of $Q_{h}$ :

$$
\left\|f(v)-Q_{h} f(v)\right\|_{0,2, k} \leq C h^{k+1}|f(v)|_{k+1,2, K} .
$$

For example, if $f\left(u_{h}\right)=u_{h}^{2}$ and $u_{h}=\sum_{i=1}^{N_{h}} c_{i} \phi_{i}$, where $\phi_{i} \in V_{h}$ is the basis satisfying $\phi_{i}\left(\xi_{j}\right)=\delta_{i j}$, where $N_{h}$ is the number of interior nodes, $\delta_{i j}$ is the symbol of Kronecker delta, for a nodal point $\xi_{j}$, then since $Q_{h} f\left(u_{h}\right)\left(\xi_{j}\right)=$ $u_{h}\left(\xi_{j}\right)^{2}=c_{j}^{2}$ we have $Q_{h} f\left(u_{h}\right)=\sum_{i=1}^{N_{h}} c_{i}^{2} \phi_{i}$. Generally,

$$
Q_{h} f\left(u_{h}\right)=\sum_{i=1}^{N_{h}} f\left(c_{i}\right) \phi_{i}
$$

for any function $f$. Note that if $V_{h}$ is the space of the lowest order, i.e., the space of order $k=0$, then $Q_{h} f\left(v_{h}\right)=f\left(v_{h}\right)$ for any $v_{h} \in V_{h}$. So from now on we consider $k \geq 1$ only. The main algorithm of this paper is as follows:

Algorithm :

- Step 1. For $n=1, \ldots, N$, on the coarse grid $\mathcal{T}_{H}$, find $\left(\hat{u}_{H}^{n}, \hat{\boldsymbol{\sigma}}_{H}^{n}\right) \in$ $V_{H} \times M_{H}$ satisfying the original nonlinear system:

$$
\begin{aligned}
\left(\alpha \hat{\boldsymbol{\sigma}}_{H}^{n}, \boldsymbol{\tau}\right)-\left(\hat{u}_{H}^{n}, \operatorname{div} \boldsymbol{\tau}\right) & =0, \quad \forall \boldsymbol{\tau} \in \boldsymbol{M}_{H}, \\
\left(\frac{\hat{u}_{H}^{n}-\hat{u}_{H}^{n-1}}{\Delta t}, v\right)+\left(\operatorname{div} \hat{\boldsymbol{\sigma}}_{H}^{n}, v\right) & =\left(Q_{H} f\left(\hat{u}_{H}^{n}\right), v\right), \quad \forall v \in V_{H} .
\end{aligned}
$$

- Step 2. For $n=1, \ldots, N$, on the fine grid $\mathcal{T}_{h}$, find $\left(\hat{u}_{h}^{n}, \hat{\boldsymbol{\sigma}}_{h}^{n}\right) \in V_{h} \times \boldsymbol{M}_{h}$ satisfying the linearized system:

$$
\left(\alpha \hat{\boldsymbol{\sigma}}_{h}^{n}, \boldsymbol{w}\right)-\left(\hat{u}_{h}^{n}, \operatorname{div} \boldsymbol{w}\right)=0, \quad \forall \boldsymbol{\tau} \in \boldsymbol{M}_{h},
$$


(12) $\left(\frac{\hat{u}_{h}^{n}-\hat{u}_{h}^{n-1}}{\Delta t}, v\right)+\left(\operatorname{div} \hat{\boldsymbol{\sigma}}_{h}^{n}, v\right)=\left(f\left(\hat{u}_{H}^{n}\right)+f_{u}\left(\hat{u}_{H}^{n}\right)\left(\hat{u}_{h}^{n}-\hat{u}_{H}^{n}\right), v\right), \forall v \in V_{h}$.

\section{Error analysis}

We define the "elliptic projection" of the solution $\left(u^{n}, \boldsymbol{\sigma}^{n}\right)$ of the problem (4)-(5) as the function $\left(\tilde{u}_{h}^{n}, \tilde{\boldsymbol{\sigma}}_{h}^{n}\right) \in V_{h} \times \boldsymbol{M}_{h}$ at each time level $n$ that satisfies the following equations:

$$
\begin{aligned}
\left(\alpha \tilde{\boldsymbol{\sigma}}_{h}^{n}, \boldsymbol{\tau}\right)-\left(\tilde{u}_{h}^{n}, \operatorname{div} \boldsymbol{\tau}\right) & =0, \quad \forall \boldsymbol{\tau} \in \boldsymbol{M}_{h}, \\
\left(\operatorname{div} \tilde{\boldsymbol{\sigma}}_{h}^{n}, v\right) & =\left(f\left(u^{n}\right)-\frac{\partial u^{n}}{\partial t}, v\right), \quad \forall v \in V_{h} .
\end{aligned}
$$

The following estimates are well known results (see [4]): for each time level $n$, $1 \leq p<\infty$,

$$
\begin{gathered}
\left\|u^{n}-\tilde{u}_{h}^{n}\right\|_{0, p} \leq C h^{r}\left\|u^{n}\right\|_{r, p}, \quad 2 \leq r \leq k+1, \\
\left\|\boldsymbol{\sigma}^{n}-\tilde{\boldsymbol{\sigma}}_{h}^{n}\right\|_{0, p} \leq C h^{r}\left\|\boldsymbol{\sigma}^{n}\right\|_{r, p}, \quad 1 \leq r \leq k+1, \\
\left\|\operatorname{div}\left(\boldsymbol{\sigma}^{n}-\tilde{\boldsymbol{\sigma}}_{h}^{n}\right)\right\|_{0, p} \leq C h^{r}\left\|\operatorname{div} \boldsymbol{\sigma}^{n}\right\|_{r, p}, \quad 1 \leq r \leq k+1 .
\end{gathered}
$$

First, we consider Step 1 (coarse grid). Let $\varepsilon^{n}:=u^{n}-\hat{u}_{H}^{n}$ and $\boldsymbol{\theta}^{n}:=\boldsymbol{\sigma}^{n}-\hat{\boldsymbol{\sigma}}_{H}^{n}$. Subtracting (9)-(10) from (4)-(5) respectively, we arrive at the following error equations:

$$
\left(\alpha \boldsymbol{\theta}^{n}, \boldsymbol{\tau}\right)-\left(\varepsilon^{n}, \operatorname{div} \boldsymbol{\tau}\right)=0, \forall \boldsymbol{\tau} \in \boldsymbol{M}_{H},
$$

$$
\left(\frac{\varepsilon^{n}-\varepsilon^{n-1}}{\Delta t}, v\right)+\left(\operatorname{div} \boldsymbol{\theta}^{n}, v\right)=\left(e^{n}, v\right)+\left(f\left(u^{n}\right)-Q_{H} f\left(\hat{u}_{H}^{n}\right), v\right), \forall v \in V_{H} .
$$

For $\eta\left(\cdot, t_{n}\right):=\eta^{n}:=u^{n}-\tilde{u}_{H}^{n}, \xi_{H}^{n}:=\tilde{u}_{H}^{n}-\hat{u}_{H}^{n}, \boldsymbol{\tau}^{n}:=\boldsymbol{\sigma}^{n}-\tilde{\boldsymbol{\sigma}}_{H}^{n}$, and $\boldsymbol{\rho}_{H}^{n}:=\tilde{\boldsymbol{\sigma}}_{H}^{n}-$ $\hat{\boldsymbol{\sigma}}_{H}^{n}$, we have $\varepsilon^{n}=\eta^{n}+\xi_{H}^{n}$ and $\boldsymbol{\theta}^{n}=\boldsymbol{\tau}^{n}+\boldsymbol{\rho}_{H}^{n}$. If we insert these decompositions into (18) and (19), then we get from (13) and (14), for $n=1, \ldots, N$,

$$
\begin{aligned}
\left(\alpha \boldsymbol{\rho}_{H}^{n}, \boldsymbol{\tau}\right)-\left(\xi_{H}^{n}, \operatorname{div} \boldsymbol{\tau}\right)= & 0, \quad \forall \boldsymbol{\tau} \in \boldsymbol{M}_{H}, \\
\left(\frac{\xi_{H}^{n}-\xi_{H}^{n-1}}{\Delta t}, v\right)+\left(\operatorname{div} \boldsymbol{\rho}_{H}^{n}, v\right)= & \left(e^{n}, v\right)+\left(f\left(u^{n}\right)-Q_{H} f\left(\hat{u}_{H}^{n}\right), v\right) \\
& -\left(\frac{\eta^{n}-\eta^{n-1}}{\Delta t}, v\right), \quad \forall v \in V_{H} .
\end{aligned}
$$

We introduce three lemmas before considering main theorems.

Lemma 3.1. If $u$ is the solution of (1), then for $e^{n}=\frac{u^{n}-u^{n-1}}{\Delta t}-\frac{\partial u^{n}}{\partial t}$,

$$
\left\|e^{n}\right\|^{2}=\frac{\Delta t}{3} \int_{(n-1) \Delta t}^{n \Delta t}\left\|\frac{\partial^{2} u}{\partial t^{2}}\right\|^{2} d t .
$$

Proof. By the Cauchy-Schwarz inequality, we see that

$$
\left\|e^{n}\right\|^{2}=\left\|\frac{\partial u^{n}}{\partial t}-\frac{u^{n}-u^{n-1}}{\Delta t}\right\|^{2}=\left\|\frac{1}{\Delta t} \int_{(n-1) \Delta t}^{n \Delta t}[t-(n-1) \Delta t] \frac{\partial^{2} u}{\partial t^{2}} d t\right\|^{2} .
$$


So,

$$
\begin{aligned}
\left\|e^{n}\right\|^{2} & \leq \frac{1}{\Delta t^{2}}\left\|\sqrt{\int_{(n-1) \Delta t}^{n \Delta t}[t-(n-1) \Delta t]^{2} d t} \sqrt{\int_{(n-1) \Delta t}^{n \Delta t}\left(\frac{\partial^{2} u}{\partial t^{2}}\right)^{2}} d t\right\|^{2} \\
& =\frac{\Delta t}{3} \int_{\Omega} \int_{(n-1) \Delta t}^{n \Delta t}\left(\frac{\partial^{2} u}{\partial t^{2}}\right)^{2} d t d x \\
& =\frac{\Delta t}{3} \int_{(n-1) \Delta t}^{n \Delta t}\left\|\frac{\partial^{2} u}{\partial t^{2}}\right\|^{2} d t .
\end{aligned}
$$

Lemma 3.2. If $u$ is the solution of (4)-(5), $\tilde{u}_{H}^{n}$ is the solution of (13)-(14) on the coarse grid $\mathcal{T}_{H}$, and $\eta\left(\cdot, t_{n}\right):=\eta^{n}:=u^{n}-\tilde{u}_{H}^{n}$, then for any $\epsilon>0$ and for $k \geq 1$,

$$
\left|\left(\eta^{n}-\eta^{n-1}, v\right)\right| \leq \frac{C H^{2 k+2}}{2 \epsilon^{2}} \int_{(n-1) \Delta t}^{n \Delta t}\left\|\frac{\partial u}{\partial t}\right\|_{k+1}^{2} d t+\frac{\Delta t \epsilon^{2}}{2}\|v\|^{2}, \quad \forall v \in V_{H} .
$$

Proof. By the Cauchy-Schwarz inequality,

$$
\begin{aligned}
\left|\left(\eta^{n}-\eta^{n-1}, v\right)\right| & =\left|\left(\int_{(n-1) \Delta t}^{n \Delta t} \frac{\partial \eta}{\partial t} d t, v\right)\right| \\
& \leq\left(\sqrt{\int_{(n-1) \Delta t}^{n \Delta t} d t} \sqrt{\int_{(n-1) \Delta t}^{n \Delta t}\left(\frac{\partial \eta}{\partial t}\right)^{2} d t},|v|\right) \\
& =\left(\sqrt{\left.\int_{(n-1) \Delta t}^{n \Delta t}\left(\frac{\partial \eta}{\partial t}\right)^{2} d t, \sqrt{\Delta t}|v|\right)}\right. \\
& \leq \frac{1}{2 \epsilon^{2}} \int_{\Omega} \int_{(n-1) \Delta t}^{n \Delta t}\left(\frac{\partial \eta}{\partial t}\right)^{2} d t d x+\frac{\Delta t \epsilon^{2}}{2}(v, v) .
\end{aligned}
$$

Interchanging the order of integration and applying (15) imply for $k \geq 1$,

$$
\begin{aligned}
\left|\left(\eta^{n}-\eta^{n-1}, v\right)\right| & \leq \frac{1}{2 \epsilon^{2}} \int_{(n-1) \Delta t}^{n \Delta t}\left\|\frac{\partial \eta}{\partial t}\right\|^{2} d t+\frac{\Delta t \epsilon^{2}}{2}(v, v) \\
& \leq \frac{C H^{2 k+2}}{2 \epsilon^{2}} \int_{(n-1) \Delta t}^{n \Delta t}\left\|\frac{\partial u}{\partial t}\right\|_{k+1}^{2} d t+\frac{\Delta t \epsilon^{2}}{2}\|v\|^{2} .
\end{aligned}
$$

The following lemma is proved easily.

Lemma 3.3. Let $g$ be a piecewise smooth on the partition $\mathcal{T}_{h}$. If $\bar{g}$ is the average value of $g(u)$ on each element $K \in \mathcal{T}_{h}$ and $\|\nabla g\|_{0, \infty} \leq B$, then

$$
|(g(u) \theta, \psi)-(\bar{g} \theta, \psi)| \leq C B h\|\theta\|_{0}\|\psi\|_{0} .
$$

Theorem 3.4. For the solution $\left(\hat{u}_{H}^{n}, \hat{\boldsymbol{\sigma}}_{H}^{n}\right) \in V_{H} \times \boldsymbol{M}_{H}$ of (9)-(10) and the solution $\left(u^{n}, \boldsymbol{\sigma}^{n}\right)$ of the equations (4)-(5), there exists a positive constant $C$ independent of $\Delta t$ and $H$ such that

$$
\left\|u^{n}-\hat{u}_{H}^{n}\right\| \leq C\left(\Delta t+H^{k+1}\right),
$$




$$
\left\|\boldsymbol{\sigma}^{n}-\hat{\boldsymbol{\sigma}}_{H}^{n}\right\| \leq C\left(\Delta t+H^{k+1}\right) .
$$

Proof. For $n=1,2, \ldots, \ell \leq N$, choosing $v=\xi_{H}^{n}$ and $\boldsymbol{\tau}=\boldsymbol{\rho}_{H}^{n}$ in (20) and (21) respectively, adding two equations, and multiplying by $\Delta t$, we obtain

$$
\begin{aligned}
& \left(\xi_{H}^{n}-\xi_{H}^{n-1}, \xi_{H}^{n}\right)+\Delta t\left(\alpha \boldsymbol{\rho}_{H}^{n}, \boldsymbol{\rho}_{H}^{n}\right) \\
= & \Delta t\left(e^{n}, \xi_{H}^{n}\right)+\Delta t\left(f\left(u^{n}\right)-Q_{H} f\left(\hat{u}_{H}^{n}\right), \xi_{H}^{n}\right)-\left(\eta^{n}-\eta^{n-1}, \xi_{H}^{n}\right) .
\end{aligned}
$$

Applying Cauchy-Schwarz inequality to the first term in left hand side and using the results of Lemma 3.1-3.2 in the right hand side, we get with $\epsilon=1$

$$
\begin{aligned}
& \frac{1}{2}\left(\xi_{H}^{n}, \xi_{H}^{n}\right)-\frac{1}{2}\left(\xi_{H}^{n-1}, \xi_{H}^{n-1}\right)+\Delta t\left(\alpha \boldsymbol{\rho}_{H}^{n}, \boldsymbol{\rho}_{H}^{n}\right) \\
\leq & \frac{1}{6} \Delta t^{2} \int_{(n-1) \Delta t}^{n \Delta t}\left\|\frac{\partial^{2} u}{\partial t^{2}}\right\|^{2} d t+\frac{1}{2} C H^{2 k+2} \int_{(n-1) \Delta t}^{n \Delta t}\left\|\frac{\partial u}{\partial t}\right\|_{k+1}^{2} d t \\
& +\Delta t\left\|\xi_{H}^{n}\right\|^{2}+\Delta t\left|\left(f\left(u^{n}\right)-Q_{H} f\left(\hat{u}_{H}^{n}\right), \xi_{H}^{n}\right)\right| .
\end{aligned}
$$

In order to bound the last term of the right-hand side of (24), we consider smoothness of $f(u)$ (i.e., assumption (A1)) and the standard approximation property of interpolation (8). Then we see that

$$
\begin{aligned}
& \left|\left(f\left(u^{n}\right)-Q_{H} f\left(\hat{u}_{H}^{n}\right), \xi_{H}^{n}\right)\right| \\
\leq & \left|\left(f\left(u^{n}\right)-f\left(\tilde{u}_{H}^{n}\right), \xi_{H}^{n}\right)\right|+\left|\left(f\left(\tilde{u}_{H}^{n}\right)-Q_{H} f\left(\tilde{u}_{H}^{n}\right), \xi_{H}^{n}\right)\right| \\
& +\left|\left(Q_{H} f\left(\tilde{u}_{H}^{n}\right)-Q_{H} f\left(\hat{u}_{H}^{n}\right), \xi_{H}^{n}\right)\right| \\
\leq & \frac{B_{1}^{2}}{2}\left\|\eta^{n}\right\|^{2}+\frac{1}{2}\left\|\xi_{H}^{n}\right\|^{2}+C H^{2 k+2} \sum_{K}\left|f\left(\tilde{u}_{H}^{n}\right)\right|_{k+1,2, K}^{2} \\
& +\frac{1}{2}\left\|\xi_{H}^{n}\right\|^{2}+\frac{B_{1}^{2}}{2}\left\|\xi_{H}^{n}\right\|^{2}+\frac{1}{2}\left\|\xi_{H}^{n}\right\|^{2} .
\end{aligned}
$$

The derivative $D^{\beta} f\left(\tilde{u}_{H}^{n}\right)$ with $|\beta|=k+1$, when expanded, yields nonlinear terms involving the derivative of $f$ up to order $k+1$ and the derivatives of $\tilde{u}_{H}^{n}$ up to order $k+1$. On the other hand from inverse inequality and the estimate (15), we have for $r=0,1, \ldots, k$ and $1 \leq p \leq \infty$,

$$
\begin{aligned}
\left\|P_{H} u^{n}-\tilde{u}_{H}^{n}\right\|_{r, p} & \leq C H^{-r-1+2 / p}\left\|P_{H} u^{n}-\tilde{u}_{H}^{n}\right\| \\
& \leq C H^{-r-1+2 / p}\left(\left\|P_{H} u^{n}-u^{n}\right\|+\left\|u^{n}-\tilde{u}_{H}^{n}\right\|\right) \\
& \leq C H^{k-r+2 / p},
\end{aligned}
$$

where $P_{H}$ is $L^{2}$-projection operator onto $V_{H}$ with well known approximation property(e.g., [2, 9]), for $r=0,1, \ldots, k, 1 \leq p \leq \infty$ and $K \in \mathcal{T}_{h}$

$$
\left\|v-P_{H} v\right\|_{r, p, K} \leq C H^{k-r+2 / p}\|v\|_{k+1,2, K}, \quad \forall v \in H^{k+1}(K) .
$$

In particular, when $r=k+1$ and $p=2$, the following holds

$$
\left\|v-P_{H} v\right\|_{k+1,2, K} \leq C\|v\|_{k+1,2, K}, \quad \forall v \in H^{k+1}(K) .
$$


Thus, for $H \leq 1$, the norm $\left\|\tilde{u}_{H}^{n}\right\|_{r, p}$ is bounded by a constant $C$ which depends on only $\left\|u^{n}\right\|_{k+1}$. Hence $\sum_{K}\left|f\left(\tilde{u}_{H}^{n}\right)\right|_{k+1,2, K}^{2}$ is bounded by a constant $C$ which is independent of $H$. Since $\alpha(x)>\alpha_{0}>0,(24)$ becomes

$$
\begin{aligned}
\left\|\xi_{H}^{n}\right\|^{2}-\left\|\xi_{H}^{n-1}\right\|^{2} \leq & \frac{\Delta t^{2}}{3} \int_{(n-1) \Delta t}^{n \Delta t}\left\|\frac{\partial^{2} u}{\partial t^{2}}\right\|^{2} d t \\
& +C H^{2 k+2} \int_{(n-1) \Delta t}^{n \Delta t}\left\|\frac{\partial u}{\partial t}\right\|_{k+1}^{2} d t+B_{1}^{2} \Delta t\left\|\eta^{n}\right\|^{2} \\
& +C \Delta t H^{2 k+2}+\left(5+B_{1}^{2}\right) \Delta t\left\|\xi_{H}^{n}\right\|^{2} .
\end{aligned}
$$

Summing above equations from $n=1, \ldots, \ell(1 \leq \ell \leq N)$, we obtain

$$
\begin{aligned}
\left\|\xi_{H}^{l}\right\|^{2} \leq & \left\|\xi_{H}^{0}\right\|^{2}+\frac{\Delta t^{2}}{3} \int_{0}^{\ell \Delta t}\left\|\frac{\partial^{2} u}{\partial t^{2}}\right\|^{2} d t+C H^{2 k+2} \int_{0}^{\ell \Delta t}\left\|\frac{\partial u}{\partial t}\right\|_{k+1}^{2} d t \\
& +B_{1}^{2} \Delta t \sum_{n=1}^{\ell}\left\|\eta^{n}\right\|^{2}+C l \Delta t H^{2 k+2}+\left(5+B_{1}^{2}\right) \Delta t \sum_{n=1}^{\ell}\left\|\xi_{H}^{n}\right\|^{2} \\
\leq & \left(1-\left(5+B_{1}^{2}\right) \Delta t\right)\left\|\xi_{H}^{0}\right\|^{2}+\frac{\Delta t^{2}}{3} \int_{0}^{\ell \Delta t}\left\|\frac{\partial^{2} u}{\partial t^{2}}\right\|^{2} d t \\
& +C H^{2 k+2} \int_{0}^{\ell \Delta t}\left\|\frac{\partial u}{\partial t}\right\|_{k+1}^{2} d t+B_{1}^{2} \Delta t \sum_{n=1}^{\ell}\left\|\eta^{n}\right\|^{2} \\
& +C T H^{2 k+2}+\left(5+B_{1}^{2}\right) \Delta t \sum_{n=0}^{\ell}\left\|\xi_{H}^{n}\right\|^{2} .
\end{aligned}
$$

When $1-\left(5+B_{1}^{2}\right) \Delta t>0$, by discrete Gronwall's lemma it follows that

$$
\begin{aligned}
\left\|\xi_{H}^{\ell}\right\|^{2} \leq C & \left(\left\|\xi_{H}^{0}\right\|^{2}+\Delta t^{2} \int_{0}^{\ell \Delta t}\left\|\frac{\partial^{2} u}{\partial t^{2}}\right\|^{2} d t+H^{2 k+2} \int_{0}^{\ell \Delta t}\left\|\frac{\partial u}{\partial t}\right\|_{k+1}^{2} d t\right. \\
& \left.+B_{1}^{2} T \max _{1 \leq n \leq \ell}\left\|\eta^{n}\right\|^{2}+H^{2 k+2}\right)
\end{aligned}
$$

In particular, let us choose the initial function $\hat{u}_{H}^{0}, \hat{\boldsymbol{\sigma}}_{H}^{0}$ to be the elliptic projection $\tilde{u}_{H}^{0}, \tilde{\boldsymbol{\sigma}}_{H}^{0}$ of the exact solution then $\xi_{H}^{0}$ vanish. Hence, the estimate $\left\|\eta^{n}\right\| \leq C H^{k+1}\left\|u^{n}\right\|_{k+1}$ for $k \geq 1$ and the assumptions (A2) on $u$ imply

$$
\left\|\varepsilon^{n}\right\| \leq\left\|\eta^{n}\right\|+\left\|\xi_{H}^{n}\right\| \leq C\left(\Delta t+H^{k+1}\right)
$$

To estimate $\boldsymbol{\theta}^{n}$, it suffices to bound $\boldsymbol{\rho}_{H}^{n}$, since $\boldsymbol{\theta}^{n}=\boldsymbol{\tau}^{n}+\boldsymbol{\rho}_{H}^{n}$ and $\boldsymbol{\tau}^{n}$ can be bounded using (16). For time level $n$ and $n-1$ in (20), we obtain the following equation:

$$
\left(\frac{\alpha\left(\boldsymbol{\rho}_{H}^{n}-\boldsymbol{\rho}_{H}^{n-1}\right)}{\Delta t}, \boldsymbol{\tau}\right)-\left(\operatorname{div} \boldsymbol{\tau}, \frac{\xi_{H}^{n}-\xi_{H}^{n-1}}{\Delta t}\right)=0, \quad \forall \boldsymbol{\tau} \in \boldsymbol{M}_{H}
$$


Taking $\boldsymbol{\tau}=\boldsymbol{\rho}_{H}^{n}$ in (26) and $v=\frac{\xi_{H}^{n}-\xi_{H}^{n-1}}{\Delta t}$ in (21) and then adding the resulting equations, we have

$$
\begin{aligned}
& \left(\frac{\alpha\left(\boldsymbol{\rho}_{H}^{n}-\boldsymbol{\rho}_{H}^{n-1}\right)}{\Delta t}, \boldsymbol{\rho}_{H}^{n}\right)+\left(\frac{\xi_{H}^{n}-\xi_{H}^{n-1}}{\Delta t}, \frac{\xi_{H}^{n}-\xi_{H}^{n-1}}{\Delta t}\right) \\
= & \left(e^{n}, \frac{\xi_{H}^{n}-\xi_{H}^{n-1}}{\Delta t}\right)+\left(f\left(u^{n}\right)-Q_{H} f\left(\hat{u}_{H}^{n}\right), \frac{\xi_{H}^{n}-\xi_{H}^{n-1}}{\Delta t}\right) \\
& -\left(\frac{\eta^{n}-\eta^{n-1}}{\Delta t}, \frac{\xi_{H}^{n}-\xi_{H}^{n-1}}{\Delta t}\right) .
\end{aligned}
$$

Let $\lambda^{n}=\frac{\xi_{H}^{n}-\xi_{H}^{n-1}}{\Delta t}$. Then multiplying above equation by $\Delta t$, we have from Cauchy-Schwarz inequality and Lemma 3.1-3.2, for $\epsilon>0$

$$
\begin{aligned}
& \frac{1}{2}\left(\alpha \boldsymbol{\rho}_{H}^{n}, \boldsymbol{\rho}_{H}^{n}\right)+\Delta t\left\|\lambda^{n}\right\|^{2} \\
\leq & \frac{1}{2}\left(\alpha \boldsymbol{\rho}_{H}^{n-1}, \boldsymbol{\rho}_{H}^{n-1}\right)+\frac{\Delta t^{2}}{6 \epsilon^{2}} \int_{(n-1) \Delta t}^{n \Delta t}\left\|\frac{\partial^{2} u}{\partial t^{2}}\right\|^{2} d t \\
& +\frac{\Delta t \epsilon^{2}}{2}\left\|\lambda^{n}\right\|^{2}+\frac{\Delta t}{2}\left\|f\left(u^{n}\right)-Q_{H} f\left(\hat{u}_{H}^{n}\right)\right\|^{2}+\frac{\Delta t}{2}\left\|\lambda^{n}\right\|^{2} \\
& +\frac{C H^{2 k+2}}{2 \epsilon^{2}} \int_{(n-1) \Delta t}^{n \Delta t}\left\|\frac{\partial u}{\partial t}\right\|_{k+1}^{2} d t+\frac{\Delta t \epsilon^{2}}{2}\left\|\lambda^{n}\right\|^{2} .
\end{aligned}
$$

The estimate (22) proved above, the boundedness of $f$ (i.e., assumption (A1)) and the standard approximation property of interpolation (8) imply that there exists a positive constant $C$ independent of $H$ such that

$$
\begin{aligned}
\left\|f\left(u^{n}\right)-Q_{H} f\left(\hat{u}_{H}^{n}\right)\right\|^{2} & \leq 2\left\|f\left(u^{n}\right)-f\left(\hat{u}_{H}^{n}\right)\right\|^{2}+2\left\|f\left(\hat{u}_{H}^{n}\right)-Q_{H} f\left(\hat{u}_{H}^{n}\right)\right\|^{2} \\
& \leq C\left(\Delta t+H^{k+1}\right)^{2} .
\end{aligned}
$$

Thus, for $\epsilon=\frac{1}{\sqrt{2}},(27)$ becomes

$$
\begin{aligned}
\frac{1}{2}\left(\alpha \boldsymbol{\rho}_{H}^{n}, \boldsymbol{\rho}_{H}^{n}\right) \leq & \frac{1}{2}\left(\alpha \boldsymbol{\rho}_{H}^{n-1}, \boldsymbol{\rho}_{H}^{n-1}\right)+\frac{\Delta t^{2}}{3} \int_{(n-1) \Delta t}^{n \Delta t}\left\|\frac{\partial^{2} u}{\partial t^{2}}\right\|^{2} d t \\
& +C H^{2 k+2} \int_{(n-1) \Delta t}^{n \Delta t}\left\|\frac{\partial u}{\partial t}\right\|_{k+1}^{2} d t+C \Delta t^{3}+C \Delta t H^{2 k+2} .
\end{aligned}
$$

Multiplying by 2 and summing from $n=1$ to $\ell(1 \leq \ell \leq N)$, we get

$$
\begin{aligned}
\left(\alpha \boldsymbol{\rho}_{H}^{\ell}, \boldsymbol{\rho}_{H}^{\ell}\right) \leq & \left(\alpha \boldsymbol{\rho}_{H}^{0}, \boldsymbol{\rho}_{H}^{0}\right)+\frac{2 \Delta t^{2}}{3} \int_{0}^{\ell \Delta t}\left\|\frac{\partial^{2} u}{\partial t^{2}}\right\|^{2} d t \\
& +C H^{2 k+2} \int_{0}^{\ell \Delta t}\left\|\frac{\partial u}{\partial t}\right\|_{k+1}^{2} d t+C \sum_{1 \leq n \leq \ell}\left(\Delta t^{3}+\Delta t H^{2 k+2}\right) .
\end{aligned}
$$


So, for $n=1, \ldots, N$,

$$
\begin{aligned}
\alpha_{0}\left\|\boldsymbol{\rho}_{H}^{n}\right\|^{2} \leq & \beta_{0}\left\|\boldsymbol{\rho}_{H}^{0}\right\|^{2}+\frac{2 \Delta t^{2}}{3} \int_{0}^{T}\left\|\frac{\partial^{2} u}{\partial t^{2}}\right\|^{2} d t \\
& +C H^{2 k+2} \int_{0}^{T}\left\|\frac{\partial u}{\partial t}\right\|_{k+1}^{2} d t+C T\left(\Delta t^{2}+H^{2 k+2}\right) .
\end{aligned}
$$

Since $\boldsymbol{\rho}_{H}^{0}$ vanishes, we obtain from (16), (25) and the assumption $(A 2)$ on $u$

$$
\left\|\boldsymbol{\theta}^{n}\right\| \leq\left\|\boldsymbol{\tau}^{n}\right\|+\left\|\boldsymbol{\rho}_{H}^{n}\right\| \leq C\left(\Delta t+H^{k+1}\right) .
$$

We now derive an error estimate for the approximate solution of the linearized system (11)-(12). Subtracting (11)-(12) from (13)-(14), we obtain the following error equations

$$
\begin{gathered}
\left(\alpha \gamma_{h}^{n}, \boldsymbol{\tau}\right)-\left(\mu_{h}^{n}, \operatorname{div} \boldsymbol{\tau}\right)=0, \quad \forall \boldsymbol{\tau} \in \boldsymbol{M}_{h}, \\
\left(\frac{\mu_{h}^{n}-\mu_{h}^{n-1}}{\Delta t}, v\right)+\left(\operatorname{div} \gamma_{h}^{n}, v\right) \\
=\left(f\left(u^{n}\right)-f\left(\hat{u}_{H}^{n}\right)-f_{u}\left(\hat{u}_{H}^{n}\right)\left(\hat{u}_{h}^{n}-\hat{u}_{H}^{n}\right), v\right) \\
+\left(\frac{\tilde{u}_{h}^{n}-\tilde{u}_{h}^{n-1}}{\Delta t}-\frac{u^{n}-u^{n-1}}{\Delta t}, v\right)+\left(e^{n}, v\right), \quad \forall v \in V_{h},
\end{gathered}
$$

where $\mu_{h}^{n}:=\tilde{u}_{h}^{n}-\hat{u}_{h}^{n}, \gamma_{h}^{n}:=\tilde{\boldsymbol{\sigma}}_{h}^{n}-\hat{\boldsymbol{\sigma}}_{h}^{n}$.

Lemma 3.5. If $\tilde{u}_{H}^{n}$ is the solution of (13)-(14) and $\hat{u}_{H}^{n}$ is the solution of (9)(10), then there exists a positive constant $C$ independent of $\Delta t$ and $H$ such that

$$
\left\|\tilde{u}_{H}^{n}-\hat{u}_{H}^{n}\right\|_{0,4} \leq C\left(H^{-1 / 2} \Delta t+H^{k+1 / 2}\right)
$$

Proof. Subtracting (9)-(10) from (13)-(14), we have

$$
\begin{gathered}
\left(\alpha \boldsymbol{\rho}_{H}^{n}, \boldsymbol{\tau}\right)-\left(\xi_{H}^{n}, \operatorname{div} \boldsymbol{\tau}\right)=0, \quad \forall \boldsymbol{\tau} \in \boldsymbol{M}_{H}, \\
\left(\frac{\xi_{H}^{n}-\xi_{H}^{n-1}}{\Delta t}, v\right)+\left(\operatorname{div} \boldsymbol{\rho}_{H}^{n}, v\right)=\left(T_{0}, v\right), \quad \forall v \in V_{H}
\end{gathered}
$$

where

$$
T_{0}:=\left\{\frac{\tilde{u}_{H}^{n}-\tilde{u}_{H}^{n-1}}{\Delta t}-\frac{\partial u^{n}}{\partial t}\right\}+\left\{f\left(u^{n}\right)-Q_{H} f\left(\hat{u}_{H}^{n}\right)\right\}:=T_{1}+T_{2} .
$$

Putting $v=\xi_{H}^{n}$ in (31) and $\boldsymbol{\tau}=\boldsymbol{\rho}_{H}^{n}$ in (30) and adding the resulting equations, we get

$$
\left(\frac{\xi_{H}^{n}-\xi_{H}^{n-1}}{\Delta t}, \xi_{H}^{n}\right)+\left(\alpha \boldsymbol{\rho}_{H}^{n}, \boldsymbol{\rho}_{H}^{n}\right)=\left(T_{1}, \xi_{H}^{n}\right)+\left(T_{2}, \xi_{H}^{n}\right) .
$$

To bound the first term of right-side of (32), we split $T_{1}$ in two terms:

$$
T_{1}=\left\{\frac{\tilde{u}_{H}^{n}-\tilde{u}_{H}^{n-1}}{\Delta t}-\frac{P_{H} u^{n}-P_{H} u^{n-1}}{\Delta t}\right\}+\left\{\frac{P_{H} u^{n}-P_{H} u^{n-1}}{\Delta t}-\frac{\partial u^{n}}{\partial t}\right\}
$$




$$
:=d_{1}+d_{2}
$$

From the well-known superconvergnce result (e.g., [11]) between the $L^{2}$-projection and the elliptic projection of $u^{n}$, we see that

$$
\begin{aligned}
\left|\left(d_{1}, \xi_{H}^{n}\right)\right| & =\left\|(\Delta t)^{-1} \int_{t_{n-1}}^{t_{n}} \frac{\partial \omega_{H}}{\partial t}(\cdot, s) d s\right\|\left\|\xi_{H}^{n}\right\| \\
& \leq(\Delta t)^{-1} \int_{t_{n-1}}^{t_{n}}\left\|\frac{\partial \omega_{H}}{\partial t}\right\| d s\left\|\xi_{H}^{n}\right\| \\
& \leq C H^{2 k+4}+\frac{1}{2}\left\|\xi_{H}^{n}\right\|^{2},
\end{aligned}
$$

where $\omega_{H}:=\tilde{u}_{H}^{n}-P_{H} u^{n}$.

From the definition of $L^{2}$-projection and Lemma 3.1, we get

$$
\begin{aligned}
\left|\left(d_{2}, \xi_{H}^{n}\right)\right|= & \mid\left(\frac{P_{H} u^{n}-P_{H} u^{n-1}}{\Delta t}-\frac{u^{n}-u^{n-1}}{\Delta t}, \xi_{H}^{n}\right) \\
& +\left(\frac{u^{n}-u^{n-1}}{\Delta t}-\frac{\partial u^{n}}{\partial t}, \xi_{H}^{n}\right) \mid \\
\leq & \frac{1}{2}\left\|e^{n}\right\|^{2}+\frac{1}{2}\left\|\xi_{H}^{n}\right\|^{2} \\
\leq & \frac{1}{6} \Delta t \int_{(n-1) \Delta t}^{n \Delta t}\left\|\frac{\partial^{2} u}{\partial t^{2}}\right\|^{2} d t+\frac{1}{2}\left\|\xi_{H}^{n}\right\|^{2} .
\end{aligned}
$$

Next, to estimate for $T_{2}$, we consider the following relation

$$
\begin{aligned}
T_{2}= & f\left(u^{n}\right)-Q_{H} f\left(\hat{u}_{H}^{n}\right) \\
= & \left(f\left(u^{n}\right)-f\left(P_{H} u^{n}\right)\right)+\left(f\left(P_{H} u^{n}\right)-f\left(\tilde{u}_{H}^{n}\right)\right) \\
& +\left(f\left(\tilde{u}_{H}^{n}\right)-f\left(\hat{u}_{H}^{n}\right)\right)+\left(f\left(\hat{u}_{H}^{n}\right)-Q_{H} f\left(\hat{u}_{H}^{n}\right)\right) .
\end{aligned}
$$

From the assumption (A1) on $f$, the well-known superconvergence result (e.g., [11]) between the $L^{2}$-projection and the elliptic projection of $u^{n}$ and the approximation property of interpolation (8), we see that for some $u^{* *} \in V$

$$
\begin{aligned}
(35) \quad\left(f\left(u^{n}\right)-f\left(P_{H} u^{n}\right), \xi_{H}^{n}\right)= & \left(f_{u}\left(P_{H} u^{n}\right)\left(u^{n}-P_{H} u^{n}\right), \xi_{H}^{n}\right) \\
& +\left(\frac{f_{u u}\left(u^{* *}\right)}{2}\left(u^{n}-P_{H} u^{n}\right)^{2}, \xi_{H}^{n}\right), \\
= & \left(\left(f_{u}\left(P_{H} u^{n}\right)-\bar{f}_{u}\left(P_{H} u^{n}\right)\right)\left(u^{n}-P_{H} u^{n}\right), \xi_{H}^{n}\right) \\
& +\left(\frac{f_{u u}\left(u^{* *}\right)}{2}\left(u^{n}-P_{H} u^{n}\right)^{2}, \xi_{H}^{n}\right), \\
(36) \quad\left|\left(f\left(P_{H} u^{n}\right)-f\left(\tilde{u}_{H}^{n}\right), \xi_{H}^{n}\right)\right| \leq & C H^{2 k+4}+\frac{1}{2}\left\|\xi_{H}^{n}\right\|^{2}, \\
(38) \quad\left|\left(f\left(\tilde{u}_{H}^{n}\right)-f\left(\hat{u}_{H}^{n}\right), \xi_{H}^{n}\right)\right| \leq & B_{1}\left\|\xi_{H}^{n}\right\|^{2},
\end{aligned}
$$


(39) $\left|\left(f\left(\hat{u}_{H}^{n}\right)-Q_{H} f\left(\hat{u}_{H}^{n}\right), \xi_{H}^{n}\right)\right| \leq C H^{2 k+2} \sum_{K}\left|f\left(\hat{u}_{H}^{n}\right)\right|_{k+1,2, K}^{2}+\frac{1}{2}\left\|\xi_{H}^{n}\right\|^{2}$.

As in the proof of Theorem 3.4, we can show with (22) that $\sum_{K}\left|f\left(\hat{u}_{H}^{n}\right)\right|_{k+1,2, K}^{2}$ is bounded by a constant $C$ which is independent of $H$. Applying Lemma 3.3 for $g\left(u^{n}\right)=f_{u}\left(P_{H} u^{n}\right)$, with the standard approximation property of $L^{2}$-projection $P_{H}$ and the assumption on $f$, we can bound each term on the right hand side of (35):

$$
\begin{aligned}
& \left|\left(f\left(u^{n}\right)-f\left(P_{H} u^{n}\right), \xi_{H}^{n}\right)\right| \\
\leq & C B_{1}\left(H\left\|u^{n}-P_{H} u^{n}\right\|\left\|\xi_{H}^{n}\right\|+\left\|u^{n}-P_{H} u^{n}\right\|_{0,4}^{2}\left\|\xi_{H}^{n}\right\|\right) \\
\leq & C H^{2 k+4}+\left\|\xi_{H}^{n}\right\|^{2} .
\end{aligned}
$$

From (33)-(40), (32) becomes

$\left\|\xi_{H}^{n}\right\|^{2}-\left\|\xi_{H}^{n-1}\right\|^{2} \leq \frac{\Delta t^{2}}{3} \int_{(n-1) \Delta t}^{n \Delta t}\left\|\frac{\partial^{2} u}{\partial t^{2}}\right\|^{2} d t+\left(6+2 B_{1}\right) \Delta t\left\|\xi_{H}^{n}\right\|^{2}+C \Delta t H^{2 k+2}$.

Summing from $n=1$ to $\ell(1 \leq \ell \leq N)$, we have

$$
\left\|\xi_{H}^{\ell}\right\|^{2}-\left\|\xi_{H}^{0}\right\|^{2} \leq \frac{\Delta t^{2}}{3} \int_{0}^{\ell \Delta t}\left\|\frac{\partial^{2} u}{\partial t^{2}}\right\|^{2} d t+\left(6+2 B_{1}\right) \Delta t \sum_{1 \leq n \leq \ell}\left\|\xi_{H}^{n}\right\|^{2}+C \ell \Delta t H^{2 k+2}
$$

Hence,

$$
\begin{aligned}
\left\|\xi_{H}^{\ell}\right\|^{2} \leq & \left(1-\left(6+2 B_{1}\right) \Delta t\right)\left\|\xi_{H}^{0}\right\|^{2}+\frac{\Delta t^{2}}{3} \int_{0}^{T}\left\|\frac{\partial^{2} u}{\partial t^{2}}\right\|^{2} d t \\
& +\left(6+2 B_{1}\right) \Delta t \sum_{0 \leq n \leq \ell}\left\|\xi_{H}^{n}\right\|^{2}+C T H^{2 k+2} .
\end{aligned}
$$

When $1-\left(6+2 B_{1}\right) \Delta t>0$, by Gronwall's lemma it follows that

$$
\left\|\xi_{H}^{\ell}\right\|^{2} \leq C\left(\left\|\xi_{H}^{0}\right\|^{2}+\Delta t^{2} \int_{0}^{T}\left\|\frac{\partial^{2} u}{\partial t^{2}}\right\|^{2} d t+H^{2 k+2}\right) .
$$

We choose the initial function $\hat{u}_{H}^{0}$ to be elliptic projection $\tilde{u}_{H}^{0}$ of the exact solution. Then $\xi_{H}^{0}=0$ and we have

$$
\left\|\xi_{H}^{n}\right\| \leq C\left(\Delta t+H^{k+1}\right)
$$

Thus, we conclude with the inverse inequality

$$
\left\|\xi_{H}^{n}\right\|_{0,4} \leq C H^{-1 / 2}\left\|\xi_{H}^{n}\right\| \leq C\left(H^{-1 / 2} \Delta t+H^{k+1 / 2}\right) .
$$

Theorem 3.6. Let $\left(\hat{u}_{h}^{n}, \hat{\boldsymbol{\sigma}}_{h}^{n}\right) \in V_{h} \times \boldsymbol{M}_{h}$ be the solution of (11)-(12). Assume that the relation between size of time step $\Delta t$ and mesh size $H$ of coarse grid satisfies $\Delta t \cdot H^{-1}=\mathcal{O}(1)$. Then there exists a positive constant $C$ independent of $\Delta t, H$ and $h$ such that

$$
\left\|u^{n}-\hat{u}_{h}^{n}\right\| \leq C\left(\Delta t+h^{k+1}+H^{2 k+1}\right),
$$




$$
\left\|\boldsymbol{\sigma}^{n}-\hat{\boldsymbol{\sigma}}_{h}^{n}\right\| \leq C\left(\Delta t+h^{k+1}+H^{2 k+1}\right) .
$$

Proof. By Taylor expansion, there exists $u^{*} \in V$ such that

$$
f\left(u^{n}\right)=f\left(\hat{u}_{H}^{n}\right)+f_{u}\left(\hat{u}_{H}^{n}\right)\left(u^{n}-\hat{u}_{H}^{n}\right)+\frac{1}{2} f_{u u}\left(u^{*}\right)\left(u^{n}-\hat{u}_{H}^{n}\right)^{2} .
$$

Hence, for any $v \in V$

$$
\begin{aligned}
& \left(f\left(u^{n}\right)-f\left(\hat{u}_{H}^{n}\right)-f_{u}\left(\hat{u}_{H}^{n}\right)\left(\hat{u}_{h}^{n}-\hat{u}_{H}^{n}\right), v\right) \\
= & \left(f_{u}\left(\hat{u}_{H}^{n}\right)\left(u^{n}-\hat{u}_{h}^{n}\right)+\frac{1}{2} f_{u u}\left(u^{*}\right)\left(u^{n}-\hat{u}_{H}^{n}\right)^{2}, v\right) .
\end{aligned}
$$

Taking $\boldsymbol{\tau}=\gamma_{h}^{n}$ in (28) and $v=\mu_{h}^{n}$ in (29) and adding two resulting equations, we have from (41)

$$
\begin{aligned}
\left(\frac{\mu_{h}^{n}-\mu_{h}^{n-1}}{\Delta t}, \mu_{h}^{n}\right)+\left(\alpha \gamma_{h}^{n}, \gamma_{h}^{n}\right)= & \left(f_{u}\left(\hat{u}_{H}^{n}\right)\left(u^{n}-\hat{u}_{h}^{n}\right)+\frac{1}{2} f_{u u}\left(u^{*}\right)\left(u^{n}-\hat{u}_{H}^{n}\right)^{2}, \mu_{h}^{n}\right) \\
& +\left(\frac{\tilde{u}_{h}^{n}-\tilde{u}_{h}^{n-1}}{\Delta t}-\frac{u^{n}-u^{n-1}}{\Delta t}, \mu_{h}^{n}\right)+\left(e^{n}, \mu_{h}^{n}\right) .
\end{aligned}
$$

Applying the assumption on $f_{u}$ and $f_{u u}$ and using the result of Lemma 3.2 and the following relationship

$$
u^{n}-\hat{u}_{h}^{n}=u^{n}-\tilde{u}_{h}^{n}+\tilde{u}_{h}^{n}-\hat{u}_{h}^{n}=u^{n}-\tilde{u}_{h}^{n}+\mu_{h}^{n},
$$

we get with the fact $\alpha>\alpha_{0}>0$

$$
\begin{aligned}
& \frac{1}{2 \Delta t}\left(\left\|\mu_{h}^{n}\right\|^{2}-\left\|\mu_{h}^{n-1}\right\|^{2}\right) \\
\leq & B_{1}^{2}\left\|u^{n}-\tilde{u}_{h}^{n}\right\|^{2}+\frac{1}{8} B_{1}^{2}\left\|\left(u^{n}-\hat{u}_{H}^{n}\right)^{2}\right\|^{2} \\
& +\frac{1}{2 \Delta t} C h^{2 k+2} \int_{(n-1) \Delta t}^{n \Delta t}\left\|\frac{\partial u}{\partial t}\right\|_{k+1}^{2} d t+\frac{1}{2}\left\|e^{n}\right\|^{2}+\left(2+B_{1}^{2}\right)\left\|\mu_{h}^{n}\right\|^{2} .
\end{aligned}
$$

Summing over $n=1$ to $\ell(1 \leq \ell \leq N)$ and multiplying by $2 \Delta t$, we have from Lemma 3.1

$$
\begin{aligned}
\left\|\mu_{h}^{\ell}\right\|^{2}-\left\|\mu_{h}^{0}\right\|^{2} \leq & \left(4+2 B_{1}^{2}\right) \Delta t \sum_{n=1}^{\ell}\left\|\mu_{h}^{n}\right\|^{2} \\
& +\Delta t B_{1}^{2} \sum_{n=1}^{\ell}\left(\left\|u^{n}-\tilde{u}_{h}^{n}\right\|^{2}+\frac{1}{4}\left\|\left(u^{n}-\hat{u}_{H}^{n}\right)^{2}\right\|^{2}\right) \\
& +C h^{2 k+2} \int_{0}^{\ell \Delta t}\left\|\frac{\partial u}{\partial t}\right\|_{k+1}^{2} d t+C \Delta t^{2} \int_{0}^{\ell \Delta t}\left\|\frac{\partial^{2} u}{\partial t^{2}}\right\|^{2} d t .
\end{aligned}
$$

When $1-\left(4+2 B_{1}^{2}\right) \Delta t>0$, by Gronwall's lemma

$$
\left\|\mu_{h}^{\ell}\right\|^{2} \leq C \Delta t \sum_{n=1}^{\ell}\left(\left\|u^{n}-\tilde{u}_{h}^{n}\right\|^{2}+\left\|\left(u^{n}-\hat{u}_{H}^{n}\right)^{2}\right\|^{2}\right)
$$




$$
+C\left(\Delta t^{2}+h^{2 k+2}+\left\|\mu_{h}^{0}\right\|^{2}\right) .
$$

If we choose the initial function $\hat{u}_{h}^{0}=\tilde{u}_{h}^{0}, \hat{\boldsymbol{\sigma}}_{h}^{0}=\tilde{\boldsymbol{\sigma}}_{h}^{0}$ then $\mu_{h}^{0}$ and $\boldsymbol{\gamma}_{h}^{0}$ vanish. It follows from (6), (15) and Lemma 3.5 that

$$
\begin{aligned}
\left\|\mu_{h}^{\ell}\right\|^{2} \leq & C \ell \Delta t \max _{1 \leq n \leq l}\left(\left\|u^{n}-\tilde{u}_{h}^{n}\right\|^{2}+\left\|u^{n}-\tilde{u}_{H}^{n}\right\|_{0,4}^{4}+\left\|\tilde{u}_{H}^{n}-\hat{u}_{H}^{n}\right\|_{0,4}^{4}\right) \\
& +C \Delta t^{2}+C h^{2 k+2} \\
\leq & C\left(\Delta t^{2}+h^{2 k+2}+H^{4(k+1)}+H^{-2} \Delta t^{4}+H^{4(k+1 / 2)}\right) .
\end{aligned}
$$

Hence, we have from the assumption $\Delta t \cdot H^{-1}=\mathcal{O}(1)$

$$
\left\|u^{n}-\hat{u}_{h}^{n}\right\| \leq\left\|u^{n}-\tilde{u}_{h}^{n}\right\|+\left\|\mu_{h}^{n}\right\| \leq C\left(\Delta t+h^{k+1}+H^{2 k+1}\right) .
$$

Now, in order to estimate $\left\|\boldsymbol{\sigma}^{n}-\hat{\boldsymbol{\sigma}}_{h}^{n}\right\|$, we consider the equation (28) for time level $n-1$ and $n$. Then we have from (29) and (41)

$$
\begin{aligned}
& \left(\frac{\alpha\left(\gamma_{h}^{n}-\gamma_{h}^{n-1}\right)}{\Delta t}, \boldsymbol{\tau}\right)-\left(\operatorname{div} \boldsymbol{\tau}, \frac{\mu_{h}^{n}-\mu_{h}^{n-1}}{\Delta t}\right)=0, \quad \forall \boldsymbol{\tau} \in M_{h}, \\
& \left(\frac{\mu_{h}^{n}-\mu_{h}^{n-1}}{\Delta t}, v\right)+\left(\operatorname{div} \gamma_{h}^{n}, v\right) \\
= & \left(f_{u}\left(\hat{u}_{H}^{n}\right)\left(u^{n}-\hat{u}_{h}^{n}\right)+\frac{1}{2} f_{u u}\left(u^{*}\right)\left(u^{n}-\hat{u}_{H}^{n}\right)^{2}, v\right) \\
& +\left(\frac{\tilde{u}_{h}^{n}-\tilde{u}_{h}^{n-1}}{\Delta t}-\frac{u^{n}-u^{n-1}}{\Delta t}, v\right)+\left(e^{n}, v\right), \quad \forall v \in V_{h} .
\end{aligned}
$$

Taking $\boldsymbol{\tau}=\gamma_{h}^{n}, v=\frac{\mu_{h}^{n}-\mu_{h}^{n-1}}{\Delta t}$ and adding the resulting equations, we obtain

$$
\begin{aligned}
& \left(\frac{\alpha\left(\gamma_{h}^{n}-\gamma_{h}^{n-1}\right)}{\Delta t}, \gamma_{h}^{n}\right)+\left(\frac{\mu_{h}^{n}-\mu_{h}^{n-1}}{\Delta t}, \frac{\mu_{h}^{n}-\mu_{h}^{n-1}}{\Delta t}\right) \\
= & \left(f_{u}\left(\hat{u}_{H}^{n}\right)\left(u^{n}-\hat{u}_{h}^{n}\right)+\frac{1}{2} f_{u u}\left(u^{*}\right)\left(u^{n}-\hat{u}_{H}^{n}\right)^{2}, \frac{\mu_{h}^{n}-\mu_{h}^{n-1}}{\Delta t}\right) \\
& +\left(\frac{\tilde{u}_{h}^{n}-\tilde{u}_{h}^{n-1}}{\Delta t}-\frac{u^{n}-u^{n-1}}{\Delta t}, \frac{\mu_{h}^{n}-\mu_{h}^{n-1}}{\Delta t}\right)+\left(e^{n}, \frac{\mu_{h}^{n}-\mu_{h}^{n-1}}{\Delta t}\right) .
\end{aligned}
$$

Letting $\lambda^{n}=\frac{\mu_{h}^{n}-\mu_{h}^{n-1}}{\Delta t}$ and multiplying by $\Delta t$, we get

$$
\begin{aligned}
& \frac{1}{2}\left(\alpha \gamma_{h}^{n}, \gamma_{h}^{n}\right)+\Delta t\left\|\lambda^{n}\right\|^{2} \\
\leq & \frac{1}{2}\left(\alpha \gamma_{h}^{n-1}, \gamma_{h}^{n-1}\right)+\Delta t\left(e^{n}, \lambda^{n}\right) \\
& +\Delta t\left|\left(f_{u}\left(\hat{u}_{H}^{n}\right)\left(u^{n}-\hat{u}_{h}^{n}\right)+\frac{1}{2} f_{u u}\left(u^{*}\right)\left(u^{n}-\hat{u}_{H}^{n}\right)^{2}, \lambda^{n}\right)\right| \\
& +\left|\left(\left(\tilde{u}_{h}^{n}-u^{n}\right)-\left(\tilde{u}_{h}^{n-1}-u^{n-1}\right), \lambda^{n}\right)\right| .
\end{aligned}
$$


So that, Cauchy-Schwarz inequality and Lemma 3.2 imply

$$
\begin{aligned}
& \frac{1}{2}\left(\alpha \gamma_{h}^{n}, \gamma_{h}^{n}\right)+\Delta t\left\|\lambda^{n}\right\|^{2} \\
\leq & \frac{1}{2}\left(\alpha \gamma_{h}^{n-1}, \gamma_{h}^{n-1}\right)+\Delta t\left(\left\|e^{n}\right\|^{2}+\frac{1}{4}\left\|\lambda^{n}\right\|^{2}\right) \\
& +\Delta t\left(B_{1}^{2}\left\|u^{n}-\hat{u}_{h}^{n}\right\|^{2}+\frac{1}{4} B_{1}^{2}\left\|\left(u^{n}-\hat{u}_{H}^{n}\right)^{2}\right\|^{2}+\frac{1}{2}\left\|\lambda^{n}\right\|^{2}\right) \\
& +C h^{2 k+2} \int_{(n-1) \Delta t}^{n \Delta t}\left\|\frac{\partial u}{\partial t}\right\|_{k+1}^{2} d t+\frac{1}{4} \Delta t\left\|\lambda^{n}\right\|^{2} .
\end{aligned}
$$

Multiplying by 2 and summing from $n=1$ to $\ell(1 \leq \ell \leq N)$, we have from Lemma 3.1 and the fact that $\gamma_{h}^{0}$ vanishes

$$
\begin{aligned}
\alpha_{0}\left\|\gamma_{h}^{\ell}\right\|^{2} \leq & \beta_{0}\left\|\gamma_{h}^{0}\right\|^{2}+C \Delta t^{2}+C h^{2 k+2} \\
& +\Delta t \sum_{n=1}^{\ell}\left(B_{1}^{2}\left\|u^{n}-\hat{u}_{h}^{n}\right\|^{2}+\frac{1}{4} B_{1}^{2}\left\|\left(u^{n}-\hat{u}_{H}^{n}\right)^{2}\right\|^{2}\right) \\
\leq & C \Delta t^{2}+C h^{2 k+2}+C \max _{1 \leq n \leq \ell}\left(\left\|u^{n}-\hat{u}_{h}^{n}\right\|^{2}+\left\|u^{n}-\hat{u}_{H}^{n}\right\|_{0,4}^{4}\right) .
\end{aligned}
$$

We conclude with (16), (42) and (15) associated with mesh size $H$ and Lemma 3.5

\begin{tabular}{|c|c|c|c|c|}
\hline$k$ & $\begin{array}{l}\text { Degree of } \\
\text { Exactness }\end{array}$ & $\begin{array}{l}\text { Barycentric } \\
\text { Coordinates }\end{array}$ & Multiplicity & Weights \\
\hline 1 & 2 & $\left(\frac{1}{2}, \frac{1}{2}, 0\right)$ & 3 & $\omega=\frac{1}{3}|K|$ \\
\hline 2 & 4 & $\begin{array}{l}\left(a_{i}, a_{i}, 1-2 a_{i}\right) \\
\quad \text { for } i=1,2 \\
a_{1}=0.445948 \\
a_{2}=0.091576\end{array}$ & 3 & $\begin{array}{c}\omega_{i} \text { for } i=1,2 \\
\omega_{1}=|K| \times 0.223382 \\
\omega_{2}=|K| \times 0.109952\end{array}$ \\
\hline
\end{tabular}

$$
\left\|\boldsymbol{\sigma}^{n}-\hat{\boldsymbol{\sigma}}_{h}^{n}\right\| \leq\left\|\boldsymbol{\sigma}^{n}-\tilde{\boldsymbol{\sigma}}_{h}^{n}\right\|+\left\|\boldsymbol{\gamma}_{h}^{n}\right\| \leq C\left(\Delta t+h^{k+1}+H^{2 k+1}\right) .
$$

As mentioned in introduction, we can get improved error estimates using the interpolation operator with the following special nodal points (Gaussian points) on triangle elements (see, for example, p. 360 in [15]):

TABLE 1. Gaussian points and weights for quadratures on a triangle of area $|K|$.

Lemma 3.7. Assume that $\mathcal{T}_{H}$ is a quasi-uniform partition of $\Omega$ into triangles. For each $K \in \mathcal{T}_{H}$ and $v \in P_{k}(K)$, there is a constant $C$, independent of $H$, such that for $k=1,2$,

$$
\left|\int_{K}\left(f(v) \xi_{H}^{n}-Q_{H} f(v) \xi_{H}^{n}\right) d x\right| \leq C H^{2 k+2}\left|f(v) \xi_{H}^{n}\right|_{2 k+1,2, K}
$$


Proof. Since the order of quadrature (degree of exactness) is $2 k, k=1,2$ for Gaussian quadrature formula on triangle and the polynomial $Q_{H} f(v) \xi_{H}^{n}$ is of degree $\leq 2 k$, by the definition of $Q_{H} f(v)$ we have

$$
\begin{aligned}
\int_{K} Q_{H} f(v) \xi_{H}^{n} d x & =\sum_{j=1}^{n_{k}} \omega_{j} Q_{H} f\left(v\left(g_{j}\right)\right) \xi_{H}^{n}\left(g_{j}\right) \\
& =\sum_{j=1}^{n_{k}} \omega_{j} f\left(v\left(g_{j}\right)\right) \xi_{H}^{n}\left(g_{j}\right) \\
& =\int_{K} Q_{H}\left(f(v) \xi_{H}^{n}\right) d x,
\end{aligned}
$$

where $\omega_{j}$ are Gaussian weights and $g_{j}$ are Gaussian points in $K$ and $n_{k}=$ $\frac{(k+1)(k+2)}{2}$ is the total number of points. Thus, the proof is completed by the error estimates of Gaussian quadrature formula (Lemma 8.4 in [15]).

Lemma 3.8. Under the assumption of Lemma 3.7, if $v \in V_{H}$ and $\|v\|_{r, p}, r=$ $0,1,2, \ldots, k, p \geq 1$ is bounded by a constant independent of $H$, then there exists a positive constant $C$ independent of $H$ such that

$$
\left|\left(f(v)-Q_{H} f(v), \xi_{H}^{n}\right)\right| \leq C H^{2 k+3}+\left\|\xi_{H}^{n}\right\|^{2} .
$$

Proof. By Lemma 3.7 we have

$$
\begin{aligned}
\left|\left(f(v)-Q_{H} f(v), \xi_{H}^{n}\right)\right| & \leq \sum_{K \in \mathcal{T}_{H}}\left|\int_{K}\left(f(v) \xi_{H}^{n}-Q_{H} f(v) \xi_{H}^{n}\right) d x\right| \\
& \leq C \sum_{K \in \mathcal{T}_{H}} H^{2 k+2}\left|f(v) \xi_{H}^{n}\right|_{2 k+1,2, K} \\
& \leq C \sum_{K \in \mathcal{T}_{H}}\left(\sum_{|\beta|=2 k+1} H^{4 k+4}\left\|D^{\beta}\left(f(v) \xi_{H}^{n}\right)\right\|_{0,2, K}^{2}\right)^{1 / 2} .
\end{aligned}
$$

Note that the product rule gives

$$
D^{\beta}\left(f(v) \xi_{H}^{n}\right)=\sum_{\gamma+\delta=\beta} \frac{\beta !}{\gamma ! \delta !} D^{\gamma} f(v) D^{\delta} \xi_{H}^{n} .
$$

So, by Hölder inequality, we get

$$
\begin{aligned}
\left\|D^{\beta}\left(f(v) \xi_{H}^{n}\right)\right\|_{0,2, K}^{2} & =\int_{K}\left|D^{\beta}\left(f(v) \xi_{H}^{n}\right)\right|^{2} d x \\
& \leq C \int_{K}\left(\sum_{\gamma+\delta=\beta}\left(D^{\gamma} f(v)\right)^{2}\right)\left(\sum_{\gamma+\delta=\beta}\left(D^{\delta} \xi_{H}^{n}\right)^{2}\right) d x
\end{aligned}
$$




$$
\leq C\left\|\sum_{\gamma+\delta=\beta}\left(D^{\gamma} f(v)\right)^{2}\right\|_{0,2, K}^{2}\left\|\sum_{\gamma+\delta=\beta}\left(D^{\delta} \xi_{H}^{n}\right)^{2}\right\|_{0,2, K}^{2},
$$

where $|\gamma|+|\delta|=2 k+1$. Hence, the equation (43) becomes

$$
\begin{aligned}
& \left|\left(f(v)-Q_{H} f(v), \xi_{H}^{n}\right)\right| \\
\leq & C \sum_{K}\left(H^{2 k+3}\|f(v)\|_{2 k+1,4, K}^{2} H^{2 k+1}\left\|\xi_{H}^{n}\right\|_{2 k+1,4, K}^{2}\right)^{1 / 2} \\
\leq & C \sum_{K}\left(\frac{1}{2 \epsilon} H^{4 k+6}\|f(v)\|_{2 k+1,4, K}^{4}+\frac{\epsilon}{2} H^{4 k+2}\left\|\xi_{H}^{n}\right\|_{2 k+1,4, K}^{4}\right)^{1 / 2} .
\end{aligned}
$$

To bound the first term of (44), we apply generalized Hölder inequality to the identity

$$
D^{\gamma}(f(v))=\sum_{i=1}^{|\gamma|}\left(\sum_{\substack{\sum_{1}^{i} \gamma_{j}=\gamma \\ \gamma_{j} \neq 0}} f^{(i)}(v) D^{\gamma_{1}} v \cdots D^{\gamma_{i}} v\right) .
$$

Then we get

$$
\begin{aligned}
& \left\|D^{\gamma}(f(v))\right\|_{0,4, K} \\
\leq & \sum_{i=1}^{|\gamma|}\left(\sum_{\substack{\sum_{1}^{i} \gamma_{j}=\gamma \\
\beta_{j} \neq 0}}\left\|f^{(i)}(v)\right\|_{L^{\infty}}\left\|D^{\gamma_{1}} v\right\|_{0, q_{1}, K} \cdots\left\|D^{\gamma_{i}} v\right\|_{0, q_{i}, K}\right) \leq C_{1},
\end{aligned}
$$

with $\sum 1 / q_{j}=1 / 4$. Here, we have used the smoothness of $f$ and the fact that $v$ is a polynomial of degree $k$ on each element and $\|v\|_{r, p}, r=0,1, \ldots, k, p \geq 1$, is bounded. Next, we consider the second term. Since $D^{\delta} \xi_{H}^{n}$ for $|\delta|>k$ is identically zero, from the inverse inequality

$$
\left\|D^{\delta} \xi_{H}^{n}\right\|_{0,4, K}^{4} \leq C_{2} H^{-4 k-2}\left\|\xi_{H}^{n}\right\|_{0,2, K}^{4}, \quad \forall|\delta| \leq k .
$$

For the constant $C$ in the equation (44), letting $\epsilon=\frac{2}{C^{2} C_{2}}$ the proof is completed.

Theorem 3.9. Assume that $\mathcal{T}_{H}$ is a quasi-uniform partition of $\Omega$ into triangles. Let $\left(\hat{u}_{h}^{n}, \hat{\boldsymbol{\sigma}}_{h}^{n}\right) \in V_{h} \times \boldsymbol{M}_{h}$ be the solution of (11)-(12). Assume that the relation between size of time step $\Delta t$ and mesh size $H$ of coarse grid satisfies $\Delta t \cdot H^{-1}=\mathcal{O}(1)$. Moreover, assume that $f$ is sufficiently smooth. Then, for $k=0,1,2$, there exists a positive constant $C$ independent of $\Delta t, H$ and $h$ such that

$$
\left\|u^{n}-\hat{u}_{h}^{n}\right\| \leq C\left(\Delta t+h^{k+1}+H^{2 k+2}\right)
$$




$$
\left\|\boldsymbol{\sigma}^{n}-\hat{\boldsymbol{\sigma}}_{h}^{n}\right\| \leq C\left(\Delta t+h^{k+1}+H^{2 k+2}\right) .
$$

Proof. In the case $k=0$, the identity

$$
Q_{h} f\left(v_{h}\right)=f\left(v_{h}\right), \quad \forall v_{h} \in V_{h}
$$

holds. This says that all terms related to interpolation disappear in the proofs of Theorem 3.4, Lemma 3.8 and Theorem 3.6. So the proof is easily completed.

In the case $k=1,2$, from the result of Lemma 3.8 the equation (39) in the proof of Lemma 3.5 becomes

$$
\left|\left(f\left(\hat{u}_{H}^{n}\right)-Q_{H} f\left(\hat{u}_{H}^{n}\right), \xi_{H}^{n}\right)\right| \leq C H^{2 k+3}+\left\|\xi_{H}^{n}\right\|^{2} .
$$

So we get

$$
\left\|\tilde{u}_{H}^{n}-\hat{u}_{H}^{n}\right\|_{0,4} \leq C\left(H^{-1 / 2} \Delta t+H^{k+1}\right),
$$

which is the improved result compared with Lemma 3.5. Using a similar argument of the proof of Theorem 3.6, we conclude the proof.

Remark 3.10. In the case $k=0$, Theorem 3.9 holds for any mixed finite element space on rectangular mesh.

\section{Numerical results}

In this section, we apply the two scale backward Euler mixed finite element method with interpolated coefficients. We perform numerical experiments to demonstrate the effect of our two scale algorithm. For this, we consider a semilinear parabolic problem:

$$
\left\{\begin{array}{rlrl}
u_{t}-\operatorname{div}(\nabla u)+u^{3}=g & & \text { in } \Omega \times[0,1) \\
u & =0 & & \text { on } \partial \Omega \times[0,1) \\
u(\cdot, 0) & =0 & & \text { in } \Omega \times\{0\},
\end{array}\right.
$$

where $\Omega=(0,1) \times(0,1)$ and $g$ is computed from the exact solution $u(t, x, y)=$ txy $(1-x)(1-y)$.

Let $\mathcal{T}_{h}$ be a uniform partition of $\Omega$ into triangles. Consider the RaviartThomas finite element space of index $1\left(R T_{1}\right)$ given as

$$
\begin{aligned}
M_{h} & :=\left\{\boldsymbol{\tau} \in H(\operatorname{div} ; \Omega):\left.\tau\right|_{K} \in R T_{1}(K), \forall K \in \mathcal{T}_{h}\right\}, \\
V_{h} & :=\left\{v \in L^{2}(\Omega):\left.v\right|_{K} \in P_{1}(K), \forall K \in \mathcal{T}_{h}\right\} .
\end{aligned}
$$

Thanks to Theorem 3.9, the bases of $P_{1}(K)$ on each triangle $K$ consist of Lagrangian nodal functions based on barycentric coordinates $\left(0, \frac{1}{2}, \frac{1}{2}\right),\left(\frac{1}{2}, 0, \frac{1}{2}\right)$ and $\left(\frac{1}{2}, \frac{1}{2}, 0\right)$. More precisely, the basis of $P_{1}(\hat{K})$ on reference triangle $\hat{K}$ is given by

$$
\{2 \xi+2 \eta-1,-2 \xi+1,-2 \eta+1\},
$$

where reference triangle $\hat{K}$ is a triangle with vertices $(0,0),(1,0)$ and $(0,1)$. Now, we apply our numerical scheme to the problem (45). We equally divide 
time interval $[0,1]$ and set $t_{n}=n \Delta t, n=1,2, \ldots, N$ and $\Delta t=1 / N$. We seek solutions at final time $t=1$.

On coarse-scale grid, our nonlinear system can be solved by an iteration method, for example:

$$
\begin{aligned}
\left(\hat{\boldsymbol{\sigma}}_{H}^{n, m}, \boldsymbol{\tau}\right)-\left(\hat{u}_{H}^{n, m}, \operatorname{div} \boldsymbol{\tau}\right) & =0, \forall \boldsymbol{\tau} \in \boldsymbol{M}_{H}, \\
\Delta t\left(\operatorname{div} \hat{\boldsymbol{\sigma}}_{H}^{n, m}, v\right)+\left(\hat{u}_{H}^{n, m}, v\right) & =\Delta t\left(g(x)-Q_{H}\left(\hat{u}_{H}^{n, m-1}\right)^{3}, v\right)+\left(\hat{u}_{H}^{n-1}, v\right), \forall v \in V_{H},
\end{aligned}
$$

where $n$ denotes the time level and $m$ the iteration index. On fine-scale grid, we solve the following linear system:

$$
\begin{aligned}
\left(\hat{\boldsymbol{\sigma}}_{h}^{n}, \boldsymbol{\tau}\right)-\left(\hat{u}_{h}^{n}, \operatorname{div} \boldsymbol{\tau}\right)= & 0, \quad \forall \boldsymbol{\tau} \in \boldsymbol{M}_{h} \\
\Delta t\left(\operatorname{div} \hat{\boldsymbol{\sigma}}_{h}^{n}, v\right)+\left(\left(1+3 \Delta t\left(\hat{u}_{H}^{n}\right)^{2}\right) \hat{u}_{h}^{n}, v\right)= & \Delta t\left(g(x)+2\left(\hat{u}_{H}^{n}\right)^{3}, v\right) \\
& +\left(\hat{u}_{h}^{n-1}, v\right), \quad \forall v \in V_{h} .
\end{aligned}
$$

For each time step, the computation of $\left(Q_{H}\left(\hat{u}_{H}^{n}\right)^{3}, \phi_{j}\right)$ for $\hat{u}_{H}^{n}=\sum c_{i} \phi_{i}$ is carried out as follows

$$
\left(Q_{H}\left(\hat{u}_{H}^{n}\right)^{3}, \phi_{j}\right)=D\left(\boldsymbol{c}^{\wedge}{ }^{\wedge}\right),
$$

where $\boldsymbol{c} .^{\wedge} 3$ is a vector whose $i$ th component is $c_{i}^{3}$ and $D$ is a diagonal matrix with $D_{i j}=\left(\phi_{i}, \phi_{j}\right)$ which is exactly computed by Gaussian quadrature rule for $k=1$ as in Table 1

$$
\left(\phi_{i}, \phi_{j}\right)=\sum_{K \in \mathcal{T}_{h}} \int_{K} \phi_{i} \phi_{j} d x=\sum_{K \in \mathcal{T}_{h}}\left(\sum_{\ell=1}^{3} \omega \phi_{i}\left(g_{\ell}\right) \phi_{j}\left(g_{\ell}\right)\right) .
$$

Thus, our two-scale product approximation procedure can lead to great computational advantages without sacrificing the overall accuracy of the scheme. Indeed, we present convergence orders for $\left\|u-\hat{u}_{h}\right\|$ and $\left\|\boldsymbol{\sigma}-\hat{\boldsymbol{\sigma}}_{h}\right\|$ in the following table. The coarse scale mesh $H$ and fine scale $h$ are chosen so that $H=h^{1 / 2}$ according to Theorem 3.9. Table 2 shows optimal convergence results which are in good agreement with the theory developed in this paper.

Table 2. $L^{2}$-error norms and convergence orders $\left(R T_{1} \times P_{1}\right.$ with $\left.\Delta t=0.1\right)$.

\begin{tabular}{|c||c|c||c|c|}
\hline $1 / h(1 / H)$ & $\left\|u-\hat{u}_{h}\right\|$ & $\left\|\boldsymbol{\sigma}-\hat{\boldsymbol{\sigma}}_{h}\right\|$ & C.O. for $u$ & C.O. for $\boldsymbol{\sigma}$ \\
\hline $4(2)$ & $1.3689 e-003$ & $5.7954 e-003$ & & \\
\hline $16(4)$ & $8.7236 e-005$ & $3.7649 e-004$ & 1.99 & 1.97 \\
\hline $36(6)$ & $1.7250 e-005$ & $7.4775 e-005$ & 2.00 & 1.99 \\
\hline $64(8)$ & $5.4596 e-006$ & $2.3704 e-005$ & 2.00 & 2.00 \\
\hline
\end{tabular}

\section{References}

[1] C. Bi and V. Ginting, Two-grid finite volume element method for linear and nonlinear elliptic problems, Numer. Math. 108 (2007), no. 2, 177-198.

[2] S. C. Brenner and L. Ridgway Scott, The Mathematical Theory of Finite Element Methods, Third edition. Texts in Applied Mathematics, 15. Springer, New York, 2008. 
[3] F. Brezzi and M. Fortin, Mixed and Hybrid Finite Element Methods, Springer-Verlag, New York, 1991

[4] Z. Chen, $L^{p}$-posteriori error analysis of mixed methods for linear and quasilinear elliptic problems, Modeling, mesh generation, and adaptive numerical methods for partial differential equations (Minneapolis, MN, 1993), 187-199, IMA Vol. Math. Appl., 75, Springer, New York, 1995.

[5] Z. Chen and J. Douglas, Approximation of coefficients in hybrid and mixed methods for nonlinear parabolic problems, Mat. Apl. Comput. 10 (1991), no. 2, 137-160.

[6] Y. Chen, Y. Huang, and D. Yu, A two-grid method for expanded mixed finite-element solution of semilinear reaction-diffusion equations, Internat. J. Numer. Methods Engrg. 57 (2003), no. 2, 193-209.

[7] C. Chen, S. Larsson, and N. Zhang, Error estimates of optimal order for finite element methods with interpolated coefficients for the nonlinear heat equation, IMA J. Numer. Anal. 9 (1989), no. 4, 507-524.

[8] I. Christie, D. F. Griffiths, A. R. Mitchell, and J. M. Sanz-Serna, Product approximation for nonlinear problems in the finite element method, IMA J. Numer. Anal. 1 (1981), no. $3,253-266$.

[9] P. G. Ciarlet, The Finite Element Method for Elliptic Problems, North-Holland, Amsterdam, New York, Oxford, 1978.

[10] C. Dawson and M. F. Wheeler, Two-grid methods for mixed finite element approximations of nonlinear parabolic equations, Domain decomposition methods in scientific and engineering computing (University Park, PA, 1993), 191-203, Contemp. Math., 180, Amer. Math. Soc., Providence, RI, 1994.

[11] J. Douglas, Jr. and J. E. Roberts, Global Estimates for Mixed Methods for second Order Elliptic Equations, Math. Comp. 44 (1985), no. 169, 39-52.

[12] J. Douglas, Jr. and T. Dupont, The effect of interpolating the coefficients in nonlinear parabolic Galerkin procedures, Math. Comp. 20 (1975), no. 130, 360-389.

[13] J. Douglas, Jr., T. Dupont, and R. E. Ewing, Incomplete iteration for time-stepping a Galerkin method for a quasilinear parabolic problem, SIAM J. Numer. Anal. 16 (1979), no. 3, 503-522.

[14] J. Douglas, Jr., R. E. Ewing, and M. F. Wheeler, The approximation of the pressure by a mixed method in the simulation of miscible displacement, RAIRO Anal. Numér. 17 (1983), no. 1, 17-33.

[15] A. Ern and J.-L. Guermond, Theory and Practice of Finite Elements, Springer-Verlag, 2004.

[16] J. Jin, S. Shu, and J. Xu, A two-grid discretization method for decoupling systems of partial differential equations, Math. Comp. 75 (2006), no. 256, 1617-1626.

[17] S. Larsson, V. Thomée, and N. Zhang, Interpolation of coefficients and transformation of the dependent variable in finite element methods for the nonlinear heat equation, Math. Meth. Appl. Sci. 11 (1989), no. 1, 105-124.

[18] W. Layton and W. Lenferink, Two-level Picard and modified Picard methods for the Navier-Stokes equations, Appl. Math. Comput. 69 (1995), no. 2-3, 263-274.

[19] D. Kim and E.-J. Park, A posteriori error estimator for expanded mixed hybrid methods, Numer. Methods Partial Differential Equations 23 (2007), no. 2, 330-349.

[20] - A Priori and a posteriori analysis of mixed finite element methods for nonlinear elliptic equations, SIAM J. Numer. Anal. 48 (2010), no. 3, 1186-1207.

[21] M.-Y. Kim, F. A. Milner, and E.-J. Park, Some observations on mixed methods for fully nonlinear parabolic problems in divergence form, Appl. Math. Lett. 9 (1996), no. $1,75-81$.

[22] M.-Y. Kim, E.-J. Park, S. G. Thomas, and M. F. Wheeler, A multiscale mortar mixed finite element method for slightly compressible flows in porous media, J. Korean Math. Soc. 44 (2007), no. 5, 1103-1119. 
[23] F. A. Milner and E.-J. Park, A mixed finite element method for a strongly nonlinear second-order elliptic problem, Math. Comp. 64 (1995), no. 211, 973-988.

[24] E.-J. Park, Mixed finite element methods for nonlinear second order elliptic problems, SIAM J. Numer. Anal. 32 (1995), no. 3, 865-885.

[25] P. A. Raviart and J. M. Thomas, A mixed finite element method for 2nd order elliptic problems, Mathematical aspects of finite element methods (Proc. Conf., Consiglio Naz. delle Ricerche (C.N.R.), Rome, 1975), 292-315. Lecture Notes in Math. 606, Springer, Berlin, 1977.

[26] J. E. Roberts and J.-M. Thomas, Mixed and hybrid methods, Handbook of numerical analysis, Vol. II, 523-639, Handb. Numer. Anal., II, North-Holland, Amsterdam, 1991.

[27] T. Russell and M. F. Wheeler, Finite element and finite difference methods for continuous flows in porous media, in The Mathematics of Reservoir Simulation, 35-106, R. E. Ewing, ed., Frontiers in Applied Mathematics 1, Society for Industrial and Applied Mathematics, Philadelphia, 1984.

[28] J. M. Sanz-Serna and L. Abia, Interpolation of the coefficients in nonlinear elliptic Galerkin procedures, SIAM J. Numer. Anal. 21 (1984), no. 1, 77-83.

[29] A. Weiser and M. F. Wheeler, On convergence of block-centered finite differences for elliptic problems, SIAM J. Numer. Anal. 25 (1988), no. 2, 351-375.

[30] L. Wu and M. B. Allen, A two-grid method for mixed finite-element solution of reactiondiffusion equations, Numer. Methods Partial Differential Equations 15 (1999), no. 3, $317-332$.

[31] J. Xu, A novel two-grid method for semilinear elliptic equations, SIAM J. Sci. Comput. 15 (1994), no. 1, 231-237.

[32] — Two-grid discretization techniques for linear and nonlinear PDEs, SIAM J. Numer. Anal. 33 (1996), no. 5, 1759-1777.

DONGHO KIM

University College

YONSEI UNIVERSITY

SEOUl 120-749, KoreA

E-mail address: dhkimm@yonsei.ac.kr

EUN-JAE PARK

Department of Mathematics AND

Department of Computational Science and EngineEring

YONSEI UNIVERSITY

SEOUl 120-749, KoreA

E-mail address: ejpark@yonsei.ac.kr

BOYOON SEO

Department of Mathematics

YONSEI UNIVERSITY

SeOul 120-749, KoreA

E-mail address: mathied@yonsei.ac.kr 